\title{
PTX3: a new mediator of bone metabolism and osteoporosis
}

\author{
Umberto Tarantino \\ Maurizio Feola \\ Monica Celi \\ Manuel Scimeca
}

Department of Orthopedics and Traumatology, "Tor Vergata" University of Rome, "Policlinico Tor Vergata" Foundation, Rome, Italy

\author{
Corresponding author: \\ Umberto Tarantino \\ Department of Orthopaedics and Traumatology, \\ "Tor Vergata", University of Rome \\ "Policlinico Tor Vergata" Foundation, \\ Via Montpellier 1 \\ 00133 Rome, Italy \\ E-mail: umberto.tarantino@uniroma2.it.
}

In the era of personalized medicine, the identification of novel mediator of bone metabolism is one of the main research fields. Indeed, the finding of new molecules involved in bone homeostasis can open new perspectives in prevention and treatment of bone degenerative age-related diseases. Osteoporosis, the most common metabolic bone disease of the elderly, presents with decreased bone mass and increased occurrence of fragility fractures ${ }^{1}$. These fractures are one of the most important problem in the elderly population, leading to significant increase of cost to health care service ${ }^{2-4}$.

Recent and contradictory data are reported about the possible role of PTX3 in bone homeostasis. Specifically, it has been described that PTX3 is able to induce the expression of Receptor Activator of Nuclear factor Kappa-B Ligand (RANKL) by osteoblasts promoting osteoclasts activation in an in vitro culture system ${ }^{5}$. On the other hand, preliminary data by Kelava et al. investigated the relationship between PTX3 expression and bone quality in mice deficient for the PTX3 gene demonstrating that PTX3 null mice had lower bone mass than their WT littermates, implying a positive involvement of PTX3 in bone formation rather than in the resorption ${ }^{6}$.

PTX3 is considered as a multifunctional glycoprotein involved in cell proliferation and migration, and deposition and remodelling of the extracellular matrix by a variety of cells such as vascular endothelial cells, smooth muscle cells, fibroblasts, adipocytes, chondrocytes, mesangial, epithelial and mesenchymal stromal cells $\mathbf{s}^{7,8}$. To establish the effective involvement of PTX3 in osteoblasts activity, and in general in bone patho-physiology, we are studying its expression and function in osteoblasts from osteoporotic patients and young subjects not affected by bone diseases. Our preliminary findings highlight the capability of human osteoblasts to express PTX3 both in vivo and in vitro models. Noteworthy, the expression of PTX3 appears significantly reduced in osteoblasts that showed a poor propensity to form hydroxyapatite crystals, especially in osteoporotic patients. The correlation between osteoblast PTX3 deficiency and low bone density that we are observing in human subject well matches with bone structure deficiency and impaired fracture healing described in the animal model of PTX3 null mice ${ }^{6}$. Specifically, Authors reported that bone regeneration requires PTX 3 for both the induction of the fracture healing process and recruitment of differentiated osteoblasts ${ }^{6}$.

Data now available about the relationship between PTX3 and bone, allow to hypothesize the mechanisms through which PTX3 could be involved in both bone metabolism and osteoporosis development (Fig. 1). In particular, PTX3 could influence osteoblasts differentiation both at level of Mesenchymal Stem Cells (MSC) and pre-osteoblasts as demonstrated by Chiellini et al. ${ }^{9}$. Therefore, in physiological conditions, the presence of PTX3 in the bone marrow environment could stimulate osteoblast differentiation and function. On the other hand, in the absence of PTX3, the balance between osteoblast and adipocyte differentiation could be shifted toward the adipocyte lineage. This phenomenon is characteristic of ultrastructural and morphological framework of osteoporotic patients, in whom the bone marrow is replaced by fat tissue. Then, PTX3 can sustain bone metabolism both increasing the number of active osteoblasts and activating osteoclasts through the RANKL/RANK pathway.

What is clear is that PTX3 is a novel regulator of bone metabolism with prominent effects on cellular process that are essential to both bone physiology and osteoporosis. All together, these considerations could lay the foundation for the development of new therapeutic approach of osteoporotic patients based on the use of human recombinant PTX3.

\section{Acknowledgments}

The Authors wish to thank Mirco Polletta for drawing Figure 1. 


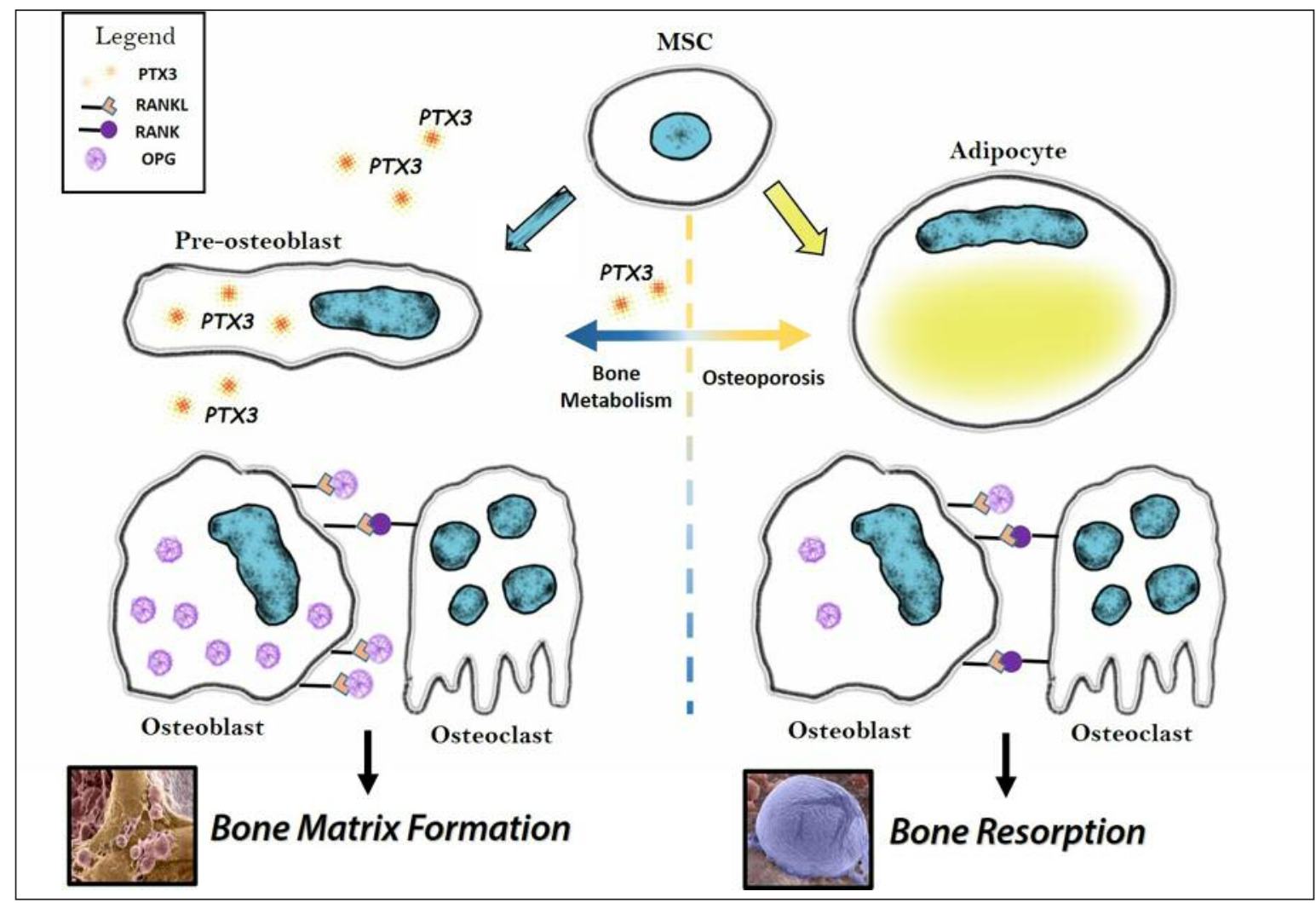

Figure 1. PTX3 as a kingmaker of bone metabolism.

\section{References}

1. Hui S, Slemenda C, Johnston C. Age and bone mass as predictors of fracture in a prospective-study. J Clin Invest. 1988; 81:1804-1809.

2. Piscitelli $P$, Tarantino $U$, Chitano $G$, et al. Updated incidence rates of fragility fractures in Italy: extension study 2002-2008. Clin Cases Miner Bone Metab. 2011;8(3):54-61.

3. Piscitelli P, Brandi ML, Tarantino U, et al. Incidence and socioeconomic burden of hip fractures in Italy: extension study 2003-2005. Reumatismo. 2010;62(2):113-118.

4. Cerocchi I, Ghera S, Gasbarra E, Feola M, Tarantino U. The clinical significance of wrist fracture in osteoporosis. Aging Clin Exp Res. 2013;25 Suppl 1:S81-82.

5. Lee EJ, et al. PTX3 stimulates osteoclastogenesis by increas- ing osteoblast RANKL production. J Cell Physiol. 2014;229 (11):1744-1752.

6. Kelava $\mathrm{T}$, Ivcevic $\mathrm{S}$, Katavic $\mathrm{V}$, et al. Increased expression of PTX3 in non-hematopoietic periosteal cells during fracture healing. Bone Abstracts. 2014;3:68.

7. Bottazzi B, Inforzato A, Messa M, et al. The pentraxins PTX3 and $S A P$ in innate immunity, regulation of inflammation and tissue remodelling. J Hepatol. 2016;64(6):1416-1427.

8. Salustri A, Garlanda C, Hirsch E, et al. PTX3 plays a key role in the organization of the cumulus oophorus extracellular matrix and in in vivo fertilization. Development. 2004;131(7):15771586.

9. Chiellini $\mathrm{C}$, et al. Characterization of human mesenchymal stem cell secretome at early steps of adipocyte and osteoblast differentiation. BMC Mol Biol. 2008;9:26. 